\title{
Strangeness in Stellar Matter
}

\author{
M. Prakash ${ }^{1}$, S. Reddy ${ }^{1}$, J. M. Lattimer ${ }^{2}$ and P. J. Ellis ${ }^{3}$ \\ ${ }^{1}$ Physics Department, \\ State University of New York at Stony Brook, \\ Stony Brook, New York-11794, USA \\ ${ }^{2}$ Earth and Space Sciences Department, \\ State University of New York at Stony Brook, \\ Stony Brook, New York-11794, USA \\ 3 School of Physics and Astronomy, \\ University of Minnesota, \\ Minneapolis, MN-55455, USA
}

\begin{abstract}
A protoneutron star is formed immediately after the gravitational collapse of the core of a massive star. At birth, the hot and high density matter in such a star contains a large number of neutrinos trapped during collapse. Trapped neutrinos generally inhibit the presence of exotic matter - hyperons, a kaon condensate, or quarks. However, as the neutrinos diffuse out in about 10-15 s, the threshold for the appearance of strangeness is reduced; hence, the composition and the structure of the star can change significantly. The effect of exotic, negatively-charged, strangeness-bearing components is always to soften the equation of state, and the possibility exists that the star collapses to a black hole at this time. This could explain why no neutron star has yet been seen in the remnant of supernova SN1987A, even though one certainly existed when neutrinos were detected on Feb. 23, 1987. With new generation neutrino detectors it is feasible to test different theoretical scenarios observationally.
\end{abstract}

\section{Introduction}

The possible existence of matter with strangeness to baryon ratio, $|S| / B$, of order unity has received a great deal of attention recently. The most likely site in which strangeness-bearing matter may exist is thought to be the interior regions of a neutron star (see Fig. 1). The physical state and internal constitution of neutron stars chiefly depends on the nature of the strong 
interactions. Although the composition and the equation of state (EOS) of neutron star matter are not yet known with certainty, QCD based effective Lagrangians have opened up intriguing possibilities. Strangeness may occur in the form of baryons, notably the $\Lambda$ and $\Sigma^{-}$hyperons, or as a Bose condensate, such as a $K^{-}$meson condensate, or in the form of strange quarks in a mixed phase of hadrons and quarks. All these possibilities involve negatively charged, strange matter, which, if present in dense matter, results in important consequences for neutron stars. For example, the appearance of strangeness-bearing components results in protoneutron (newly born) stars having larger maximum masses than catalyzed (older, neutrino-free) neutron stars, a reversal from ordinary nucleons-only matter. This permits the existence of metastable protoneutron stars that could collapse to black holes during their deleptonization. This scenario is outlined in Sec. 4 (for further details see Refs. [1, 2]).

\section{Some Observed Neutron Star Properties}

To date, the observations relevant to the understanding of neutron star composition, structure, and evolution are mainly those of masses and surface temperatures.

\section{$2.1 \quad$ Masses}

Observed neutron star masses are shown in Fig. 2. The smallest range that is consistent with all of the data has an upper limit of $1.44 M_{\odot}$ from PSR1913+16 and a lower limit of $1.36 M_{\odot}$ from the precisely measured total mass of PSR $2127+11 \mathrm{C}$ and its companion. The upper limit, $M=1.44 M_{\odot}$, provides constraints on the neutron star EOS. A conservative estimate of this upper limit is shown by the dashed line at $M=1.5 M_{\odot}$. Any neutron star EOS has to support a maximum mass of at least this value.

The fact that all the measured neutron star masses consistently lie within a narrow range of $1.4 M_{\odot}$ has raised many intriguing issues. One possible explanation stems from evolutionary arguments. Since neutron stars are formed in the gravitational core collapse of massive stars, their masses may depend on the structure of the progenitor star. The cores of stars which evolve into neutron stars have precollapse masses of about $1.4 M_{\odot}$, which introduces a natural mass scale for possible neutron star masses. The final neutron star mass may, however, depend on the amount of accretion at 
times subsequent to a neutron star's birth. Thus, rigorous arguments for the happenstance of observations are not yet available. Later we will return to the question of whether or not it is the nature of strong interactions which restricts the stars to this mass range.

\subsection{Thermal emissions and surface temperatures}

Neutron stars are born with interior temperatures of order 20-50 MeV, but cool via neutrino emission to temperatures of less than $1 \mathrm{MeV}$ within minutes [5]. The subsequent cooling consists of two phases: a neutrinodominated cooling epoch followed by a photon-dominated cooling epoch. The temperature and luminosity of this thermal radiation is controlled by the evolution of the interior temperature of the star. In the standard scenario, in which the cooling occurs by the so-called modified Urca processes, $n+n \rightarrow n+p+e^{-}+\bar{\nu}_{e}$ and $n+p+e^{-} \rightarrow n+n+\nu_{e}$, the interior cooling is slow enough that the surface temperatures of neutron stars remain above $10^{6} \mathrm{~K}$ for about $10^{5} \mathrm{yr}$, and they are potentially observable for this length of time in the X-ray or UV bands. Recently, it has been pointed out [6] that a rapid cooling may occur via the direct Urca processes, $n \rightarrow p+e^{-}+\bar{\nu}_{e}$ and $p+e^{-} \rightarrow n+\nu_{e}$, and similar processes involving muons if the proton fraction in super-nuclear matter reaches values in excess of about 10\%. This occurs when exotic matter, such as a pion or a kaon condensate or quark matter, is present. Similar direct Urca processes involving strangeness-bearing hyperons also lead to rapid cooling [7]. In this case the core cools so rapidly that a temperature inversion develops. The size of the cooler interior grows as the energy from the hot crust is conducted to the core. After about 1 to 100 yr, depending on the stars's structure, this cooling wave reaches the surface, and the surface temperature plummets to very low values. Thus, observations of surface temperatures hold the potential of revealing the internal constitution of neutron stars $[8,9]$.

Through recent advances in imaging X-ray telescopes, it has been possible to detect X-rays from some 14 rotation-powered neutron stars (see, for example, Ref. [10]). Figure 3 shows the inferred surface temperatures of some X-ray emitters. Of these, four appear to have the signatures of neutron stars on the initial cooling curve. These pulsars [Vela (PSR 0833-45), Geminga (PSR 0630+18) and PSR's 0656+14, 1055-52] span an age bracket of $10^{4}$ to $5 \times 10^{5} \mathrm{yr}$. Their X-ray luminosities are currently being compared with calculations for both the standard cooling and the rapid cooling sce- 
narios. The surface temperature of a pulsar may not be entirely determined by the cooling of the neutron star, since irradiation of its surface by magnetopsheric X-rays [11] could also play a role. Thus, all temperature estimates or "measurements" must be considered as upper limits.

\section{$3 \quad$ Neutron Star Structure}

In hydrostatic equilibrium, the structure of a spherically symmetric neutron star is determined by the Tolman-Oppenheimer-Volkov (TOV) equations [12]:

$$
\begin{aligned}
& \frac{d M(r)}{d r}=4 \pi r^{2} \epsilon(r) \\
& \frac{d P(r)}{d r}=-\frac{G M(r) \epsilon(r)}{c^{2} r^{2}} \frac{\left[1+\frac{P(r)}{\epsilon(r)}\right]\left[1+\frac{4 \pi r^{3} P(r)}{M(r) c^{2}}\right]}{\left[1-\frac{2 G M(r)}{c^{2} r}\right]} .
\end{aligned}
$$

Above, $G$ is the gravitational constant, $P$ is the pressure, $\epsilon$ is the energy density inclusive of the rest mass density, and $M(r)$ is the enclosed gravitational mass. The quantity $R_{s}=2 G M / c^{2}$ is known as the Schwarzschild radius. The gravitational and baryon masses of the star are defined by

$$
\begin{aligned}
& M_{G} c^{2}=\int_{0}^{R} d r 4 \pi r^{2} \epsilon(r) \\
& M_{A} c^{2}=m_{A} \int_{0}^{R} d r 4 \pi r^{2} \frac{n(r)}{\left[1-\frac{2 G M(r)}{c^{2} r}\right]^{1 / 2}},
\end{aligned}
$$

where $m_{A}$ is the baryonic mass and $n(r)$ is the baryon number density. The binding energy of the star is then B.E. $=\left(M_{A}-M_{G}\right) c^{2}$.

By specifying the EOS of enclosed matter, $P=P(\epsilon)$, the structure of the star is determined by choosing a central pressure $P_{c}=P\left(\epsilon_{c}\right)$ at $r=0$ and integrating the coupled differential equations (11) and (2) out to the star surface at $r=R$ determined by the condition $P(r=R)=0$. The significance of general relativity may be gauged by the magnitude of $R_{s} / R$. When $R_{s} / R \ll 1$, the structure is essentially determined by Newtonian gravity. The surface approaches the event horizon as $R_{s} / R \rightarrow 1$; larger values result in black hole configurations. 


\subsection{Macroscopic properties}

A comparison of the results obtained by solving the TOV equations for two different EOS's which differ in their high density behavior offers useful insights concerning the physical attributes of a star. Figure 4 shows some of these features. The top left panel contains $P$ vs. $\epsilon$ for two representative EOS's. The dash-dotted curve shows the causal EOS $P=\epsilon$. The solid curve is referred to as the soft EOS, since the pressure varies less rapidly with the energy density than the dashed curve, which is termed the stiff EOS. The EOS directly influences the physical attributes of a star. For example, the limiting or maximum mass for the stiff EOS is larger than that for the soft EOS. This is seen from the mass versus radius plots (top right panel) and also from the mass versus central baryon density (center left panel). Also, the stiffer the EOS, the lower is the central density of the maximum mass star. Further, the radius (top right panel) and the binding energy (center right panel) of the maximum mass star are larger for the stiff EOS than for the soft EOS. Finally, the bottom panels contrast the moments of inertia (bottom left) and the surface red shift (bottom right) for the soft and stiff EOS's. Stellar configurations with central densities $n_{c}>n_{c}\left(M_{\max }\right)$ are unstable towards small perturbations, since the gravitational attraction in such stars overwhelms the repulsive forces in matter. Such stars are thought to form black holes. Stars with central densities $n_{c}<n_{c}\left(M_{\max }\right)$ represent stable configurations and are candidates for stable neutron stars.

\subsection{Constraints on the equation of state}

Stringent constraints may be placed on the EOS if measurements of stars' masses, radii, pulsar frequencies, moments of inertia, etc. are available. To see how this works, it is useful to consider the dependence of some of these properties on the mass and radius in a so-called $M-R$ diagram. We turn now to describe the basic elements of such an exercise.

1. The denominator in Eq. (2) requires that the radius $R>R_{s}=$ $2 G M / c^{2}$. This yields $M / M_{\odot} \leq R / R_{s}(\odot)$, where $R_{s}(\odot)=2 G M_{\odot} / c^{2} \cong$ $2.95 \mathrm{~km}$.

2. By requiring the pressure in the center of the star to be finite, $P_{c}<$ $\infty$, Weinberg [13] has shown that $R>(9 / 8) R_{s}$. This translates to $M / M_{\odot} \leq(8 / 9) R / R_{s}(\odot)$. 
3. The condition that the adiabtic sound speed $c_{s}=(d P / d \epsilon)^{1 / 2} \leq c$, where $c$ is the speed of light, leads to the condition that $R>1.39 R_{s}$, giving $M / M_{\odot} \leq R /\left(1.39 \times R_{s}(\odot)\right)$.

4. If, instead of employing the causal EOS $P=\epsilon$ at all densities, one requires it to hold above a fiducial density $n_{t} \cong 2 n_{0}$ (below which the EOS is presumed to be known), the limit $R>1.52 R_{s}$ is obtained [14, yielding $M / M_{\odot} \leq R /\left(1.52 \times R_{s}(\odot)\right)$.

Notice that the above restrictions follow from general principles. In practice, however, these conditions allow a large class of EOS's to be consistent with observations. One is thus forced to utilize additional constraints that are based on observations. This procedure, however, is necessarily time dependent insofar as future observations may alter the precise limits! In the hope that continued measurements will indeed prescribe such limits, we will note the constraints employed currently.

1. The limiting or maximum mass $M_{\max }$ should exceed the largest of the observed neutron star masses. Currently, this condition is taken to imply $M_{\max } \geq 1.44 M_{\odot}$, the most accurately measured neutron star mass [3, 4].

2. Nearly all (up to 99\%) of the binding energy of a neutron star is released in the form of neutrinos during the birth of a neutron star, after the gravitational core collapse of a massive $\left(\geq 8 M_{\odot}\right)$ star results in a type II supernova. Estimates [5] of the energy released in neutrinos from the SN 1987A explosion lie in the range $(2-4) \times 10^{53}$ ergs. This places a restriction on the EOS that the B.E $\geq(2-4) \times 10^{53}$ ergs.

3. The Keplerian frequency of the star (this is the rotational frequency $\Omega_{K}$ beyond which the star will begin to shed mass at the equator) should exceed the spin period of the fastest spinning pulsar, namely that of PSR $1957+20$. This translates to $P_{K} \geq 1.56 \mathrm{~ms}$. (In reality, a star may spin at a frequency lower than $\Omega_{K}$ due to viscous effects. Choosing the larger Keplerian frequency thus gives an upper limit.) General relativistic calculations of rapidly rotating stars give 14]

$$
\Omega_{K} \cong 7.7 \times 10^{3}\left(M_{\max } / M_{\odot}\right)^{1 / 2}\left(R_{\max } / 10 \mathrm{~km}\right)^{-3 / 2} \mathrm{~s}^{-1},
$$

where $M_{\max }$ and $R_{\max }$ refer to the mass and radius of the non-rotating spherical configuration. It is worthwhile to note that the discovery of 
a sub-millisecond pulsar (say of $0.5 \mathrm{~ms}$ ), as was purported [15] to be the case in the wake of the SN1987A explosion and later retracted [16], would place rather severe limits on the EOS.

4. Another limit [17, 18] employs the maximum moment of inertia, expressed in terms of $M_{\max }$ and $R_{\max }$ of the non-rotating configurations:

$$
I_{\max }=0.6 \times 10^{45} \frac{\left(M_{\max } / M_{\odot}\right)\left(R_{\max } / 10 \mathrm{~km}\right)^{2}}{1-0.295\left(M_{\max } / M_{\odot}\right) /\left(R_{\max } / 10 \mathrm{~km}\right)} \mathrm{g} \mathrm{cm}^{2} .
$$

Precise measurements of $I$ would severely limit the allowed region in the $M-R$ plane.

In Fig. 5, the implications of the various restrictions mentioned above are considered. Besides the theoretical constraints, observational constraints imposed by mass, moments of inertia, and pulsar periods are illustrated. Also shown are the mass-radius relationships of two representative EOS's. To date, data are consistent with a wide variety of EOS's, which highlights the need for continuing observations.

\section{The Fate of a Newborn Neutron Star}

After a supernova explosion, the gravitational mass of the remnant is less than $1 \mathrm{M}_{\odot}$. It is lepton rich and has an entropy per baryon of $S \simeq 1$ (in units of Boltzmann's constant $k_{B}$ ). The leptons include both electrons and neutrinos, the latter being trapped in the star because their mean free paths in the dense matter are of order $1 \mathrm{~cm}$, whereas the stellar radius is about 15 $\mathrm{km}$. Accretion onto the neutron star increases its mass to the $1.3-1.5 \mathrm{M}_{\odot}$ range, and should mostly cease after a second. It then takes about 10-15 s [5] for the trapped neutrinos to diffuse out, and in the diffusion process they leave behind most of their energy, heating the protoneutron star to fairly uniform entropy values of about $S=2$. Cooling continues as thermallyproduced neutrinos diffuse out and are emitted. After about $50 \mathrm{~s}$, the star becomes completely transparent to neutrinos, and the neutrino luminosity drops precipitously [19].

Denoting the maximum mass of a cold, catalyzed neutron star by $M_{\max }$ and the maximum mass of the protoneutron star with abundant trapped leptons by $M_{\max }^{L}$, there are two possible ways that a black hole could form after a supernova explosion. First, accretion of sufficient material could 
increase the remnant's mass to a value greater than either $M_{\max }$ or $M_{\max }^{L}$ and produce a black hole, which then appears on the accretion time scale [20]. Second, if exotic matter plays a role and if accretion is insignificant after a few seconds, then for $M_{\max }^{L}>M>M_{\max }$, where $M$ is the final remnant mass, a black hole will form as the neutrinos diffuse out 11, 21, 22, 23, 24 on the deleptonization time scale of $10-15 \mathrm{~s}$.

The existence of metastable neutron stars has some interesting implications. First, it could explain why no neutron star is readily apparent in the remnant of SN1987A despite our knowledge that one existed until at least $12 \mathrm{~s}$ after the supernova's explosion (see Sec. 6). Second, it would suggest that a significant population of relatively low mass black holes exists [25], one of which could be the compact object in the X-ray binary $4 \mathrm{U} 1700-37$ 近.

How is the stellar structure, particularly the maximum mass, influenced by the trapped neutrinos? (The finite entropy plays a lesser role [1].) In order to investigate this question, one needs the EOS up to $\sim 10$ times the baryon density encountered in the center of a nucleus. The EOS at such high densities is not known with any certainty. Nevertheless, recent work [26, 27, 28] has emphasized the possibility that hyperons, a condensate of $K^{-}$mesons, or $u, d$, and $s$ quarks, may be present in addition to nucleons and leptons. These additional components can appear separately or in combination with one another. Compared to a star containing just plain-vanilla nucleons and leptons, the presence of these additional components qualitatively changes the way in which the structure of the star depends upon neutrino trapping [1].

\subsection{Equilibrium conditions}

The composition of the star is constrained by three important physical principles: baryon conservation, charge neutrality and beta equilibrium. The third exists because the time scales of weak interactions, including those of strangeness-violating processes, are short compared to the dynamical time scales of evolution (see Sec. 5). For example, the process $p+e^{-} \leftrightarrow n+\nu_{e}$ in equilibrium establishes the relation

$$
\mu \equiv \mu_{n}-\mu_{p}=\mu_{e}-\mu_{\nu_{e}}
$$

allowing the proton chemical potential to be expressed in terms of three independent chemical potentials: $\mu_{n}, \mu_{e}$, and $\mu_{\nu_{e}}$. 
At densities where $\mu$ exceeds the muon mass, muons can be formed by $e^{-} \leftrightarrow \mu^{-}+\bar{\nu}_{\mu}+\nu_{e}$, hence the muon chemical potential is

$$
\mu_{\mu}=\mu_{e}-\mu_{\nu_{e}}+\mu_{\nu_{\mu}}
$$

requiring the specification of an additional chemical potential $\mu_{\nu_{\mu}}$. However, unless $\mu>m_{\mu} c^{2}$, the net number of $\mu$ 's and $\nu_{\mu}$ 's per baryon (designated by $Y_{\mu}$ and $Y_{\nu_{\mu}}$, respectively) is zero, because no muon-flavor leptons are present at the onset of trapping, so $Y_{\nu_{\mu}}=-Y_{\mu}$ determines $\mu_{\nu_{\mu}}$. Following deleptonization, $Y_{\nu_{\mu}}=0$, and $Y_{\mu}$ is determined by $\mu_{\mu}=\mu_{e}$ for $\mu_{e}>m_{\mu} c^{2}$, or by $Y_{\mu}=0$ otherwise.

Negatively charged kaons can be formed in the process $n+e^{-} \leftrightarrow n+$ $K^{-}+\nu_{e}$ when $\mu_{K^{-}}=\mu$ becomes equal to the energy of the lowest eigenstate of a $K^{-}$in matter. In addition, weak reactions for the $\Lambda, \Sigma$, and $\Xi$ hyperons are all of the form $B_{1}+\ell \leftrightarrow B_{2}+\nu_{\ell}$, where $B_{1}$ and $B_{2}$ are baryons, $\ell$ is a lepton, and $\nu_{\ell}$ is a neutrino of the corresponding flavor. The chemical potential for a baryon $B$ with baryon number $b_{B}$ and electric charge $q_{B}$ is then given by the general relation

$$
\mu_{B}=b_{B} \mu_{n}-q_{B} \mu
$$

which leads to

$$
\mu_{\Lambda}=\mu_{\Sigma^{0}}=\mu_{\Xi^{0}}=\mu_{n} \quad ; \quad \mu_{\Sigma^{-}}=\mu_{\Xi^{-}}=\mu_{n}+\mu \quad ; \quad \mu_{p}=\mu_{\Sigma^{+}}=\mu_{n}-\mu .
$$

The same considerations apply to quarks, for which Eq. (9) gives

$$
\mu_{d}=\mu_{s}=\left(\mu_{n}+\mu\right) / 3 \quad ; \quad \mu_{u}=\left(\mu_{n}-2 \mu\right) / 3 .
$$

Therefore, if there are no trapped neutrinos present, so that $\mu_{\nu_{\ell}}=0$, there are two independent chemical potentials $\left(\mu_{n}, \mu_{e}\right)$ representing conservation of baryon number and charge. If trapped neutrinos are present $\left(\mu_{\nu_{\ell}} \neq 0\right)$, further constraints, due to conservation of the various lepton numbers over the dynamical time scale of evolution, must be specified. At the onset of trapping, during the initial inner core collapse, $Y_{\ell}=Y_{e}+Y_{\nu_{e}} \approx 0.4$ and $Y_{e} / Y_{\nu_{e}} \sim 5-7$, depending upon the density [29]. These numbers are not significantly affected by variations in the EOS. Following deleptonization, $Y_{\nu_{e}}=0$, and $Y_{e}$ can vary widely, both with the density and EOS.

As long as both weak and strong interactions are in equilibrium, the above general relationships determine the constituents of the star during its 
evolution. Since electromagnetic interactions give negligible contributions, it is sufficient to consider the non-interacting (Fermi gas) forms for the partition functions of the leptons. Hadrons, on the other hand, receive significant contributions at high density from the less well known strong interactions.

\subsection{Neutrino-poor versus neutrino-rich stars}

A detailed discussion of the composition and structure of protoneutron stars may be found in Refs. [1, 2]. The main findings were that the structure depends more sensitively on the compostion of the star than its entropy and that the trapped neutrinos play an important role in determining the composition. Since the structure is chiefly determined by the pressure of the strongly interacting constituents and the nature of the strong interactions is poorly understood at high density, several models of dense matter, including matter with strangeness-rich hyperons, a kaon condensate and quark matter were studied there. For the purpose of illustration, we show here only the cases in which hyperons and quarks appear at high densities. The qualitative trends when other forms of strangeness, including kaon condensates, appear are very similar.

Figure 6 shows the various concentrations, $Y_{i}$ (the number of particles of species $i$ per baryon), as a function of density when the only hadrons allowed are nucleons. The arrows indicate the central density of the maximum mass stars. The left hand panel refers to the case in which the neutrinos have left the star. At high density the proton concentration is about $30 \%$, charge neutrality ensuring an equal number of negatively charged leptons. This relatively large value is the result of the symmetry energy increasing nearly linearly with density in this model. Many non-relativistic potential models [30] predict a maximum proton concentration of only $10 \%$. However, the generic results discussed here are not sensitive to the behavior of the nuclear symmetry energy. The effects of neutrino trapping are displayed in the right hand panel. The fact that $\mu_{\nu_{e}} \neq 0$ in Eq. (7) results in larger values for $\mu_{e}$ and $Y_{e}$. Because of charge neutrality, $Y_{p}$ is also larger, and it approaches $40 \%$ at high density. It is clear that the maximum mass configuration has a much lower density when neutrinos are trapped than when they are not. As is evident from the bottom row of Table 1, neutrino-trapping reduces the maximum mass $M_{\text {max }}^{L}$ from the value found in neutrino-free matter $M_{\text {max }}$; although neutrino-trapped nucleons-only matter contains more leptons and 
more leptonic pressure, it also contains more protons and, therefore, less baryonic pressure. Thermal effects increase the pressure and therefore the maximum mass, but only slightly. Even for $S=2$, the central temperature is only $\sim 50 \mathrm{MeV}$, which is much less than the nucleon Fermi energies. Thus, because $M_{\max }^{L} \lesssim M_{\max }$, a black hole could only form promptly after bounce from nucleons-only stars, in the absence of significant accretion at late times.

Table 1. Maximum masses of stars with baryonic matter that undergoes a phase transition to quark matter without $\left(Y_{\nu}=0\right)$ and with $\left(Y_{L e}=0.4\right)$ trapped neutrinos. Results are for a mean field model of baryons and a bag model of quarks. $B$ denotes the bag pressure in the quark EOS.

\begin{tabular}{c|c|c|c|c}
\multicolumn{4}{c}{$M_{\max } / M_{\odot}$} \\
\hline \hline & \multicolumn{2}{|c|}{ Without hyperons } & \multicolumn{2}{c}{ With hyperons } \\
\hline $\begin{array}{c}B \\
\left(\mathrm{MeV} \mathrm{fm}^{-3}\right)\end{array}$ & $Y_{\nu}=0$ & $Y_{L e}=0.4$ & $Y_{\nu}=0$ & $Y_{L e}=0.4$ \\
\hline 136.6 & 1.440 & 1.610 & 1.434 & 1.595 \\
150 & 1.444 & 1.616 & 1.436 & 1.597 \\
200 & 1.493 & 1.632 & 1.471 & 1.597 \\
250 & 1.562 & 1.640 & 1.506 & 1.597 \\
\hline No quarks & 1.711 & 1.645 & 1.516 & 1.597 \\
\hline \hline
\end{tabular}

For comparison, Fig. 7 shows the compositions of neutrino-free matter (left panel) and neutrino-trapped matter (right panel) in the event that strange particles are allowed to appear. In neutrino-free matter, one expects that the $\Lambda$, with a mass of $1116 \mathrm{MeV}$, and the $\Sigma^{-}$, with a mass of $1193 \mathrm{MeV}$, both first appear at roughly the same density, because the somewhat higher mass of the $\Sigma^{-}$is compensated by the presence of $\mu_{e}$ in the equilibrium condition of the $\Sigma^{-}$. More massive and more positively charged particles than these appear at higher densities. Notice that with the appearance of the negatively charged $\Sigma^{-}$hyperon, which competes with the leptons in maintaining charge neutrality, the lepton concentrations begin to fall. When quarks appear, at around $4 n_{0}$ (for $B=200 \mathrm{MeV} \mathrm{fm}^{-3}$ ), the neutral and negative particle abundances begin to fall, since quarks furnish both negative charge and baryon number. 
Trapped neutrinos again increase the proton and electron abundances, and this strongly influences the threshold for the appearance of hyperons. The $\Lambda$ and the $\Sigma$ 's now appear at densities higher than those found in the absence of neutrinos. In addition, the transition to a mixed phase with quarks is delayed to about $10 n_{0}$, which is beyond the central density of the maximum mass star (see arrow in Fig. 7). This makes the overall EOS stiffer, so that when matter contains strangeness the behavior is opposite to that of the nucleons-only case. Specifically, the maximum mass is larger in the neutrino-trapped case. This behavior is summarized in Table 1 for different assumptions about the composition and was first noted by Thorsson, Prakash and Lattimer [21] in the study of stars with kaon condensates and subsequently by Keil and Janka [22] of stars with hyperons.

Evolutionary calculations [5, 22] without accretion show that it takes on the order of 10-15 s for the trapped neutrino fraction to vanish for a nucleons-only EOS (see also Sec. 5). To see qualitatively what might transpire during the early evolution, we show in Fig. 8 the dependence of the maximum stellar mass upon the trapped neutrino fraction $Y_{\nu_{e}}$, which decreases during the evolution. When the only hadrons are nucleons (np), the maximum mass increases with decreasing $Y_{\nu_{e}}$, whereas when hyperons $(\mathrm{npH})$ or kaons $(\mathrm{npK})$ are also present, it decreases. Further, the rate of decrease accelerates for rather small values of $Y_{\nu_{e}}$. Coupled with this is the fact that the central density of stars will tend to increase during deleptonization. The implication is clear. If hyperons, kaons, or other negatively-charged hadronic species are present, an initially stable star can change into a black hole after most of the trapped neutrinos have left, and this takes $10-15 \mathrm{~s}$. This happens only if the remnant mass $M$ satisfies $M_{\max }^{L}>M>M_{\max }$.

It must be emphasized that the maximum mass of the cold catalyzed star still remains uncertain due to the uncertainty in strong interactions at high density. At present, all nuclear models can only be effectively constrained at nuclear density and by the condition of causality at high density. The resulting uncertainty is evident from the range of possible maximum masses predicted by the different models. Notwithstanding this uncertainty, our findings concerning the effects of neutrino trapping offer intriguing possibilities for distinguishing between the different physical states of matter. These possibilities include both black hole formation in supernovae and the signature of neutrinos to be expected from supernovae. 


\section{$5 \quad$ Evolution Time Scales}

The evolution of the star will be governed by the time scale for neutrino diffusion, whether the star is in the deleptonizing or cooling phase. These time scales depend on the EOS, which determines the stellar size, and the neutrino opacities. The composition and temperature in the central regions of the star at the beginning of deleptonization are characterized by a high lepton fraction $\left(Y_{L e}=0.4\right)$ and low entropy per baryon $(S \sim 1)$, while the cooling phase is characterized by $Y_{\nu_{e}} \sim 0$ and $S \sim 2$. A full treatment of neutrino transport during the evolution requires opacities for a wide range of composition and matter degeneracy. The dynamical changes in the lepton fraction and the temperature modify the composition of matter and the typical neutrino energies in the inner core. Roughly, the neutrino diffusion time scale is proportional to $R^{2}(c \lambda)^{-1}$, where $R$ is the stellar radius and $\lambda$ is the appropriate effective neutrino mean free path. In Ref. [1], the time scales in the two major evolutionary phases were estimated for nucleonsonly matter, using semi-analytical considerations. In the deleptonization phase, the diffusion time is dominated by charged current interactions and was found to be

$$
\tau_{d} \simeq \frac{9 R^{2}}{19 c \lambda_{a b s}^{o}} \simeq \frac{44.3}{f}\left(\frac{R}{10 \mathrm{~km}}\right)^{2} \mathrm{~s},
$$

where $\lambda_{a b s}^{o}$ is the fiducial absorption mean free path at the expected initial $\nu_{e}$ energy, $E_{\nu_{e}} \simeq 260 \mathrm{MeV}$. The factor $f=3-10$ accounts for degeneracy and interaction effects and is not yet well-determined. This leads to a deleptonization time of 5-15 s, in agreement with more detailed numerical simulations. The result in Eq. (12) shows clearly how the deleptonization time depends on the EOS through the radius of the star, $R$, and the opacity. Similarly, the thermal cooling time, in which neutral current interactions are also important, was estimated to be $\tau_{c}=(1-2) \tau_{d}$.

It is of some importance to contrast these dynamical time scales with the weak interaction time scales controlling the onset of strangeness-bearing components. We have seen earlier that strangeness is most likely to appear near the end of deleptonization. During this epoch, the central temperatures are expected to be high $(T=10-30 \mathrm{MeV})$. The time scale for the onset of strangeness in the form of a kaon condensate was recently addressed by Muto et al. [31, who considered the time evolution of the condensate amplitude $\theta(t)$, but only at $T=1 \mathrm{MeV}$, which would be appropriate for 
a cold catalyzed star. They found that the onset of strangeness was of the same order as the cooling time, which would have major implications for the neutrino luminosity of a cooling neutron star. The relevant weak interaction rates are, however, extremely sensitive to temperature. Figure 9 shows the time evolution of the condensate amplitude close to the threshold density for temperatures in the range 1-10 $\mathrm{MeV}$. It is evident that, for temperatures appropriate for the cooling phase, the weak interaction time scales are much smaller than the duration of the cooling phase. Therefore, to a very good approximation, the appearance of a kaon condensate may be considered as instantaneous. The onset of strangeness in the form of hyperons and/or quarks is likely to be similarly rapid.

\section{Supernova SN1987A}

On February 23 of 1987, neutrinos were observed [32] from the explosion of supernova SN1987A, indicating that a neutron star, not a black hole, was initially present. (The appearance of a black hole would have caused an abrupt cessation of any neutrino signal [19].) The neutrino signal was observed for a period of at least $12 \mathrm{~s}$, after which counting statistics fell below measurable limits. From the handful of events observed, only the average neutrino energy, $\sim 10 \mathrm{MeV}$, and the total binding energy release of $\sim(0.1-0.2) \mathrm{M}_{\odot}$ could be estimated.

These estimates, however, do not shed much light on the composition of the neutron star. This is because, to lowest order, the average neutrino energy is fixed by the neutrino mean free path in the outer regions of the protoneutron star. Further, the binding energy exhibits a universal relationship [1] for a wide class of EOS's, including those with strangeness bearing components, namely

$$
\text { B.E. }=(0.065 \pm 0.01)\left(M_{B} / \mathrm{M}_{\odot}\right)^{2} \mathrm{M}_{\odot},
$$

where $M_{B}$ is the baryonic mass. This allows us only to determine a remnant gravitational mass of $(1.14-1.55) \mathrm{M}_{\odot}$, but not the composition.

The ever-decreasing optical luminosity (light curve) [33] of the remnant of SN1987A suggests two arguments against the continued presence of a neutron star. First, accretion onto a neutron star at the Eddington limit is already ruled out for the usual hydrogen-dominated Thomson electron scattering opacity. (However, if the atmosphere surrounding the remnant contains a sufficient amount of iron-like elements, as Chen and Colgate [34 
suggest, the appropriate Eddington limit is much lower.) Second, a Crab-like pulsar cannot exist in SN1987A, since the emitted magnetic dipole radiation would be observed in the light curve. Either the magnetic field or the spin rate of the neutron star remnant would have to be much less than in the case of the Crab and what is inferred from other young neutron stars. The spin rate of a newly formed neutron star is expected to be high; however, the time scale for the generation of a significant magnetic field is not well known and could be greater than 10 years.

Although most of the binding energy is released during the initial accretion and collapse stage in about a second after bounce, the neutrino signal continued for a period of at least $12 \mathrm{~s}$. The compositionally-induced changes in the structure of the star occur on the deleptonization time scale, which we have estimated to be of order 10-15 s [1], not on the binding energy release time scale. Thus, the duration of the neutrino signal from SN1987A was comparable to the time required for the neutrinos initially trapped in the star to leave. However, counting statistics prevented measurement of a longer duration, and this unfortunate happenstance prevents one from distinguishing a model in which negatively-charged matter appears and a black hole forms from a less exotic model, in which a neutron star still exists. As we have pointed out, the maximum stable mass drops by as much as $0.2 \mathrm{M}_{\odot}$ when the trapped neutrinos depart if negatively charged particles are present, which could be enough to cause collapse to a black hole.

Observed neutron stars lie in a very small range of gravitational masses (see Fig. 2). Thielemann et al. [35] and Bethe and Brown [36] have estimated the gravitational mass of the remnant of SN1987A to be in the range $(1.40-1.56) \mathrm{M}_{\odot}$, using arguments based on the observed amounts of ejected ${ }^{56} \mathrm{Ni}$ and/or the total explosion energy. This range extends above the largest accurately known value for a neutron star mass, $1.44 \mathrm{M}_{\odot}$, so the possibility exists that the neutron star initially produced in SN1987A could be unstable in the cold, deleptonized state. In this case, SN1987A would have become a black hole once it had deleptonized, and no further signal would be expected. Should this scenario be observationally verified, it would provide strong evidence for the appearance of strange matter.

\section{$7 \quad$ Future Directions}

The emitted neutrinos, of all flavors, are the only direct probe of the mechanism of supernova explosions and the structure of newly formed neutron 
stars. The cooling of the star can yield information on the stellar composition. The two most important microphysical ingredients for detailed simulations of the cooling of a newborn neutron star are the EOS of dense matter and the neutrino opacities.

Future efforts must address the crucial question of the strong interactions of strange particles in dense matter - even near nuclear equilibrium density, our knowledge is sketchy at present.

Surprisingly little attention has been paid to the effects of composition and of strong interactions of the ambient matter on neutrino opacities. It is essential that the opacities be consistent with the composition, which has not been a feature of protoneutron star models to date. A first effort in this direction, including the presence of strangeness in the form of hyperons, has revealed significant modifications of the neutrino opacities [37]. Results from this study on neutral-current neutrino cross sections are shown in Fig. 10.

Significant contribution to the neutrino opacity arises from scattering involving $\Sigma^{-}$hyperons. Although the lowest order tree level contributions from the neutral $\Lambda$ and $\Sigma^{0}$ vanish (due to their zero weak hyper-charge), these particles, when present, furnish baryon number, which decreases the relative concentrations of nucleons. This leads to significant reductions in the opacity. The neutrino cross sections depend sensitively on the Fermi momenta and effective masses of the various particles present in matter. As long as one or the other hyperon is present, the cross sections are significantly modified from the case of nucleons-only matter.

In neutrino-trapped matter, the appearance of negatively charged hyperons $\left(\Sigma^{-}\right)$is delayed to higher densities (relative to neutrino-free matter); also, their abundances are suppressed. However, the presence of neutral hyperons, such as the $\Lambda$, results in neutrino abundances that grow with density. This leads to large enhancements in the cross sections for neutrinos (of characteristic energies close to the local neutrino chemical potential) compared to those in normal nucleonic matter.

Modifications due to strangeness in the cooling phase are somewhat different than in the deleptonization phase. In the cooling phase, in which matter is nearly neutrino free, the response of the $\Sigma^{-}$hyperons to thermal neutrinos is significant. However, the presence of a large number of $\Lambda$ hyperons, to which the neutrinos do not couple, decreases the total cross sections relative to nucleonic matter.

These findings suggest several directions for further study. These in- 
clude extension to consider correlations between the different particles and RPA corrections. The presence of charged particles, such as the $\Sigma^{-}$, could make available low energy collective plasma modes through electromagnetic correlations, in addition to the scalar, vector, and iso-vector correlations. Calculations of neutrino opacities from charged current reactions (which are important during the deleptonization phase), in strangeness-rich matter whose constituents exhibit varying degrees of degeneracy, are required for a complete description of the evolution.

What can be expected in future detections? In an optimistic scenario, several thousand neutrinos from a typical galactic supernova might be seen in upgraded neutrino detectors, such as SNO in Canada and Super Kamiokande in Japan. (For rough characteristics of present and future neutrino detectors, see Ref. [38].) Among the interesting features that could be sought are:

1. Possible cessation of a neutrino signal, due to black hole formation.

2. Possible burst or light curve feature associated with the onset of negatively-charged, strongly interacting matter near the end of deleptonization, whether or not a black hole is formed.

3. Identification of the deleptonization/cooling epochs by changes in luminosity evolution or neutrino flavor distribution.

4. Determination of a radius-mean free path correlation from the luminosity decay time or the onset of neutrino transparency.

5. Determination of the neutron star mass from the universal binding energy-mass relation.

\section{Acknowledgement}

We dedicate this article to Gerry Brown in honor of his 70th birthday. We gratefully acknowledge collaborations with I. Bombaci, Manju Prakash, R. Knorren, and J. R. Cooke. This work was supported by the U. S. Dept. of Energy under grants DOE/DE/FG02-87ER-40388, 40328, and 40317, and by the NASA grant NAG52863. 


\section{References}

[1] M. Prakash, I. Bombaci, Manju Prakash, P.J. Ellis, J.M. Lattimer and R. Knorren, nucl-th/9603042, Phys. Rep. (1996) in press.

[2] P.J. Ellis, J.M. Lattimer and M. Prakash, Comments on Nucl. and Part. Phys. (1996) in press.

[3] S.E. Thorsett, Z. Arzoumanian, M.M. McKinnon and J.H. Taylor, Astrophys. J. 405 (1994) L29; M. H. van Kerkwijk, J. van Paradijs and E. J. Zuiderwijk, Astron. \& Astrophys. 303 (1995) 497.

[4] G.E. Brown, J.C. Weingartner and R.A.M.J. Wijers, Astrophys. J. 463 (1996) 297.

[5] A. Burrows and J.M. Lattimer, Astrophys. J. 307 (1986) 178; A. Burrows, Ann. Rev. Nucl. Sci. 40 (1990) 181.

[6] J.M. Lattimer, C.J. Pethick, M. Prakash, and P. Haensel, Phys. Rev. Lett. 66 (1991) 2701.

[7] Madappa Prakash, Manju Prakash, J.M. Lattimer and C.J. Pethick, Astrophys. J. Lett. 390 (1992) L77.

[8] C.J. Pethick, Rev. Mod. Phys. 64 (1992) 1133.

[9] M. Prakash, Phys. Rep. 242 (1994) 297.

[10] H. Ögelman, in The Lives of the Neutron Stars, M.A. Alpar, Ü. Kiziloğlu and J. van Paradijs (eds), (Dordrecht: Kluwer) 1995.

[11] J.P. Halpern and M. Ruderman, Astrophys. J. 415 (1993) 286.

[12] R.C. Tolman, Proc. Nat. Acad. Sci. USA 20 (1934) 3; J.R. Oppenheimer and G.M. Volkoff, Phys. Rev. 55 (1939) 374.

[13] S. Weinberg, Gravitation and Cosmology: Principles and Applications of the General Theory of Relativity (New York: Wiley) 1972.

[14] J.M. Lattimer, M. Prakash, D. Masak and A. Yahil, Astrophys. J. 355 (1990) 241.

[15] C. Kristian, et al., Nature 338 (1989) 234.

[16] C. Pennypacker et al., private communication (1990).

[17] P. Haensel, Copernicus Astronomical Center Preprint (1990).

[18] C.J. Pethick, D.G. Ravenhall and C.P. Lorenz, Nucl. Phys. A584 (1995) 675.

[19] A. Burrows, Astrophys. J. 334 (1988) 891.

[20] G.E. Brown, S.W. Bruenn and J.C. Wheeler, Comments Astrophys. 16 (1992) 153. 
[21] V. Thorsson, M. Prakash and J.M. Lattimer, Nucl. Phys. A572 (1994) 693.

[22] W. Keil and H.T. Janka, Astron. \&6 Astrophys. 296 (1994) 145.

[23] M. Prakash, J. Cooke and J.M. Lattimer, Phys. Rev. D52 (1995) 661.

[24] N.K. Glendenning, Astrophys. J. 448 (1995) 797.

[25] G.E. Brown and H.A. Bethe, Astrophys. J. 423 (1994) 659.

[26] N.K. Glendenning, Nucl. Phys. A493 (1989) 521; J. Ellis, J.I. Kapusta and K.A. Olive, Nucl. Phys. B348 (1991) 345.

[27] D.B. Kaplan and A.E. Nelson, Phys. Lett. B175 (1986) 57; B179 (1986) 409(E).

[28] N.K. Glendenning, Phys. Rev. D46 (1992) 1274.

[29] H.A. Bethe, G.E. Brown, J. Applegate and J.M. Lattimer, Nucl. Phys. A324 (1979) 487.

[30] R.B. Wiringa, V. Fiks and A. Fabrocini, Phys. Rev. C38 (1988) 1010.

[31] T. Muto, T. Tatsumi and N. Iwamoto, Kyoto preprint KUNS-1382 (1996).

[32] K. Hirata et al., Phys. Rev. Lett. 58 (1987) 1490; R.M. Bionta et al., Phys. Rev. Lett. 58 (1987) 1494.

[33] S. Kumagai, T. Sigeyama, M. Hashimoto and K. Nomoto, Astron. 86 Astrophys. 243 (1991) L13.

[34] K. Chen and S.A. Colgate, Los Alamos preprint LA-UR-95-2972 (1995).

[35] F.-K. Thielemann, M. Hashimoto and K. Nomoto, Astrophys. J. 349 (1990) 222.

[36] H.A. Bethe and G.E. Brown, Astrophys. J. 445 (1995) L129.

[37] S. Reddy and M. Prakash, Astrophys. J. (1996) in press.

[38] A. Burrows, D. Klein and R. Gandhi, Phys. Rev. D45 (1992) 3361. 


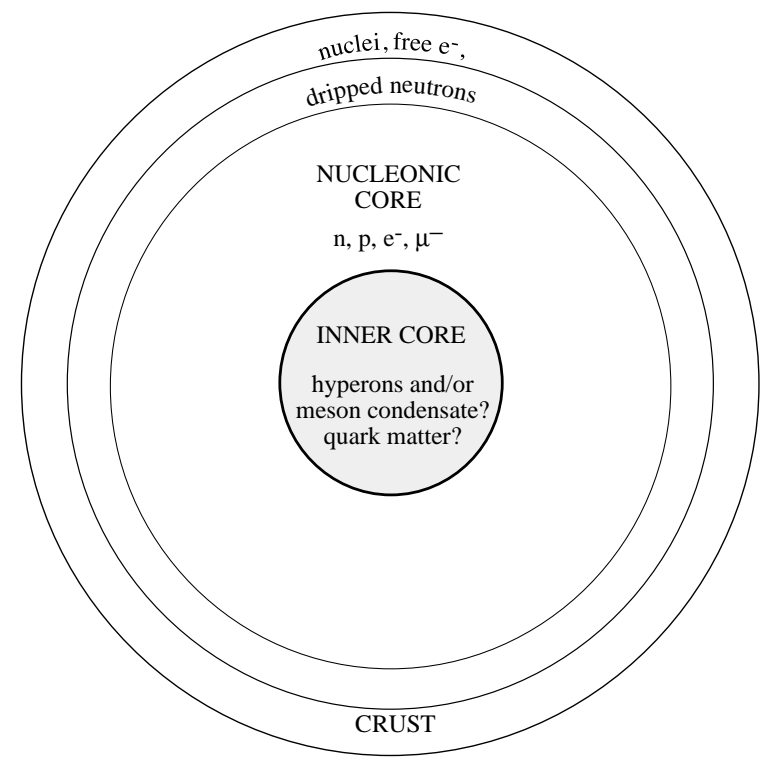

Fig.1. Schematic cross-sectional view of a neutron star. 


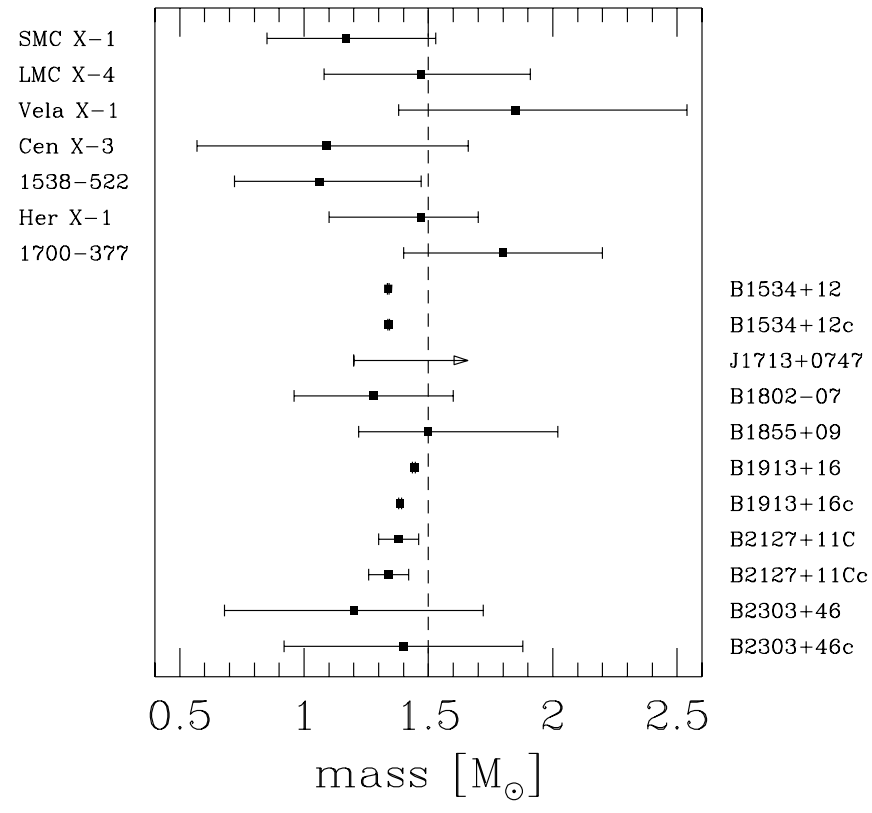

Fig.2. Measured neutron star masses from Refs. [3, 4]. The upper seven values are for X-ray pulsars, the lower eleven for radio pulsars and their companions. Error bars indicate $95 \%$ confidence limits. 


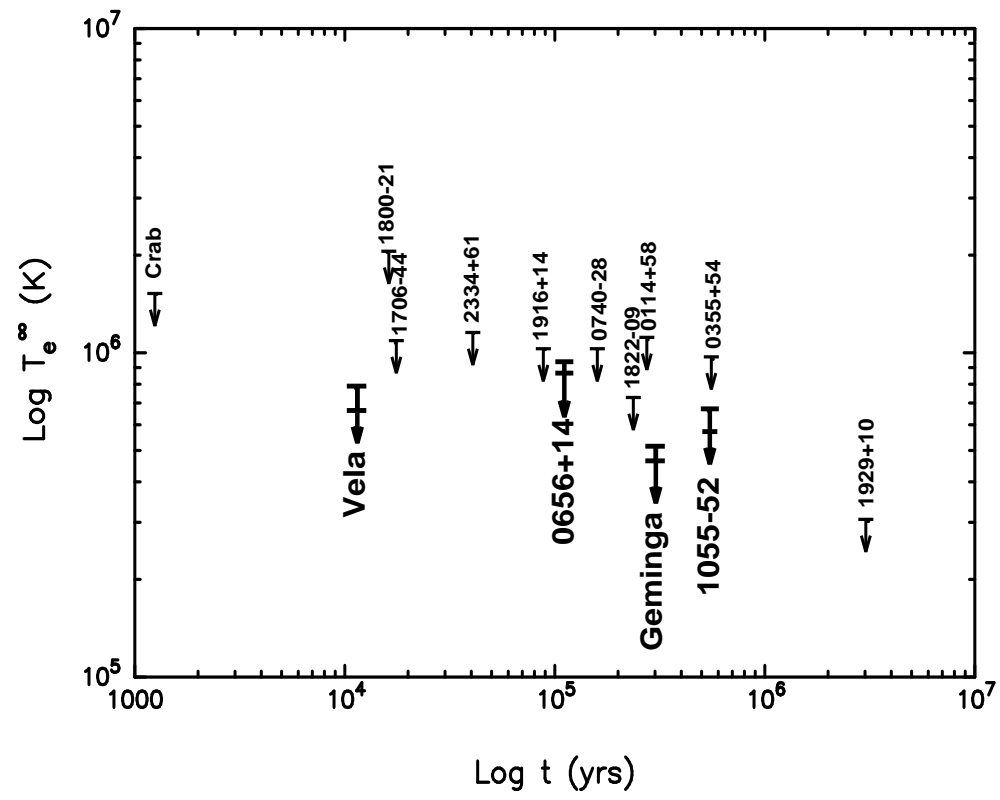

Fig.3. Measurement, estimates, or upper limits for the surface temperature of fourteen pulsars 10]. (Figure after Dany Page.) The plotted ages are the pulsar spin-down ages. 

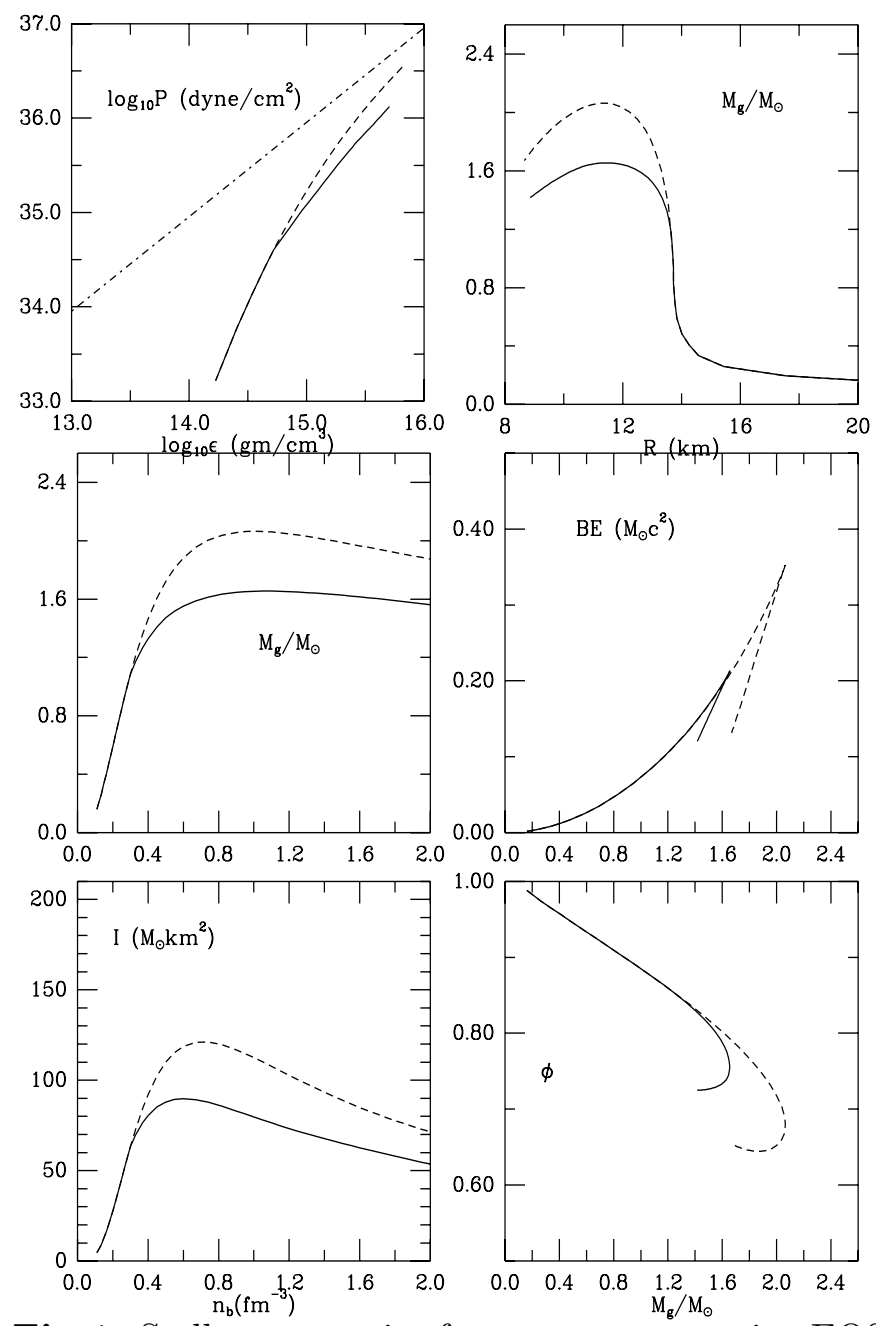

Fig.4. Stellar properties for two representative EOS's. 


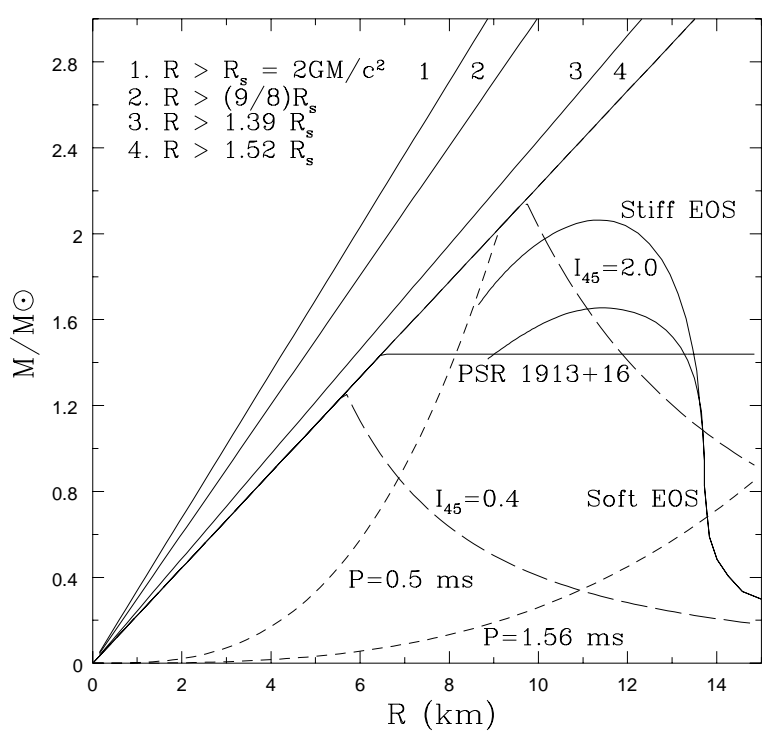

Fig.5. Valid EOS's must produce neutron stars which lie to the right of curve labeled 4 . Current observational constraints include $M>M_{P S R 1913+16}, I>0.4 \times 10^{45} \mathrm{~g} \mathrm{~cm}^{2}$ (Crab pulsar), and $P_{\min }<1.6 \mathrm{~ms}$ (PSR 1957+20). The promise of future observations is illustrated with curves of other values of $I$ and $P$. 

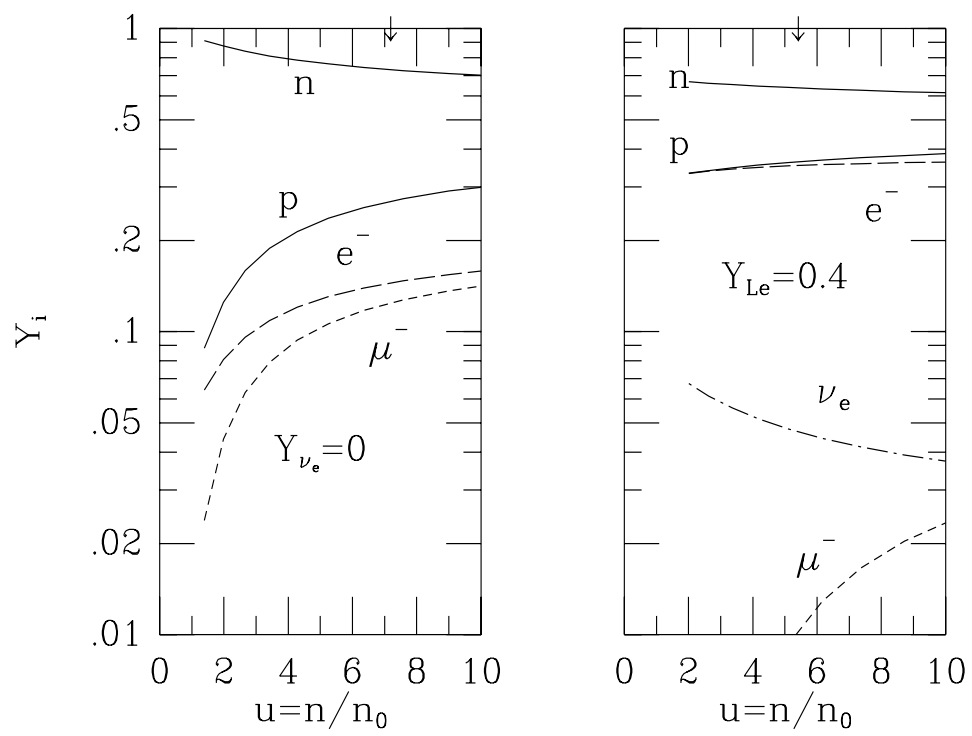

Fig.6. Individual concentrations, $Y_{i}$, as a function of the baryon density ratio $u=n / n_{0}$, where $n_{0}$ is the density of equilibrium nuclear matter. The arrows indicate the central density of the maximum mass stars. Left panel: neutrino free. Right panel: with trapped neutrinos $\left(Y_{\ell}=0.4\right)$. 

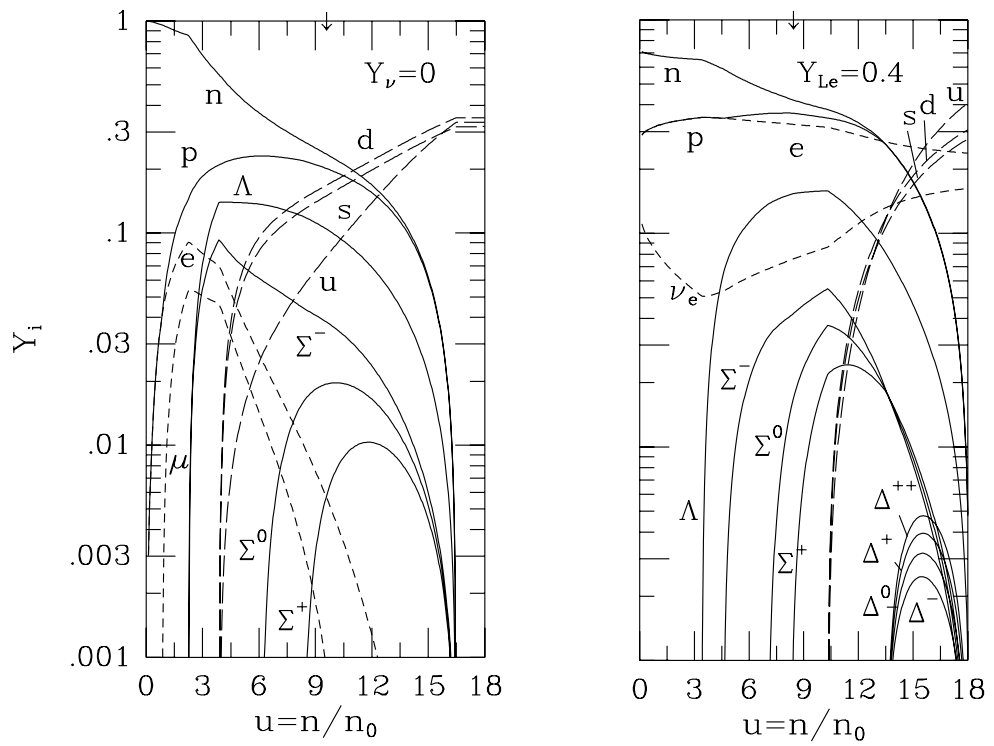

Fig.7. As for Fig. 6, but for matter which contains strangeness-bearing hyperons and quarks, as well as nucleons and leptons. 


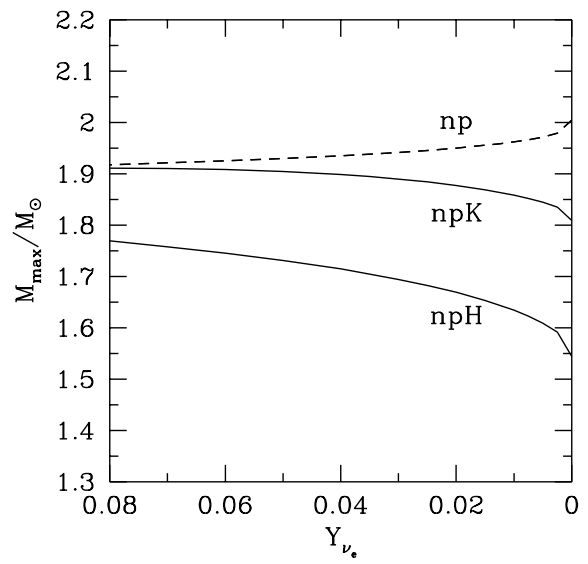

Fig.8. Maximum neutron star mass as a function of $Y_{\nu_{e}}$ for hadronic matter with only nucleons (np) or with nucleons and hyperons $(\mathrm{npH})$ or kaons $(\mathrm{npK})$. 


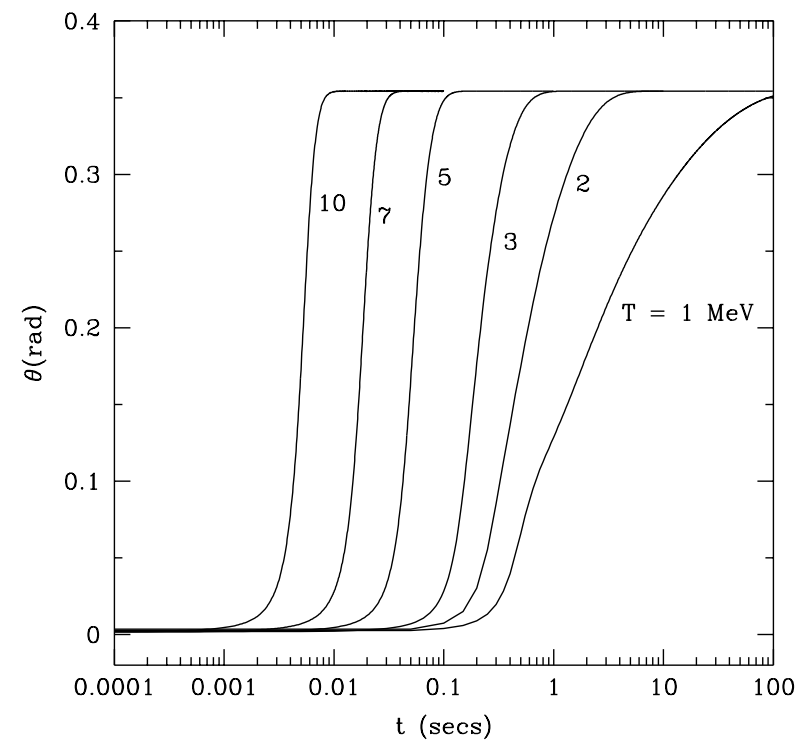

Fig.9. Kaon condensate amplitude as a function of time. 

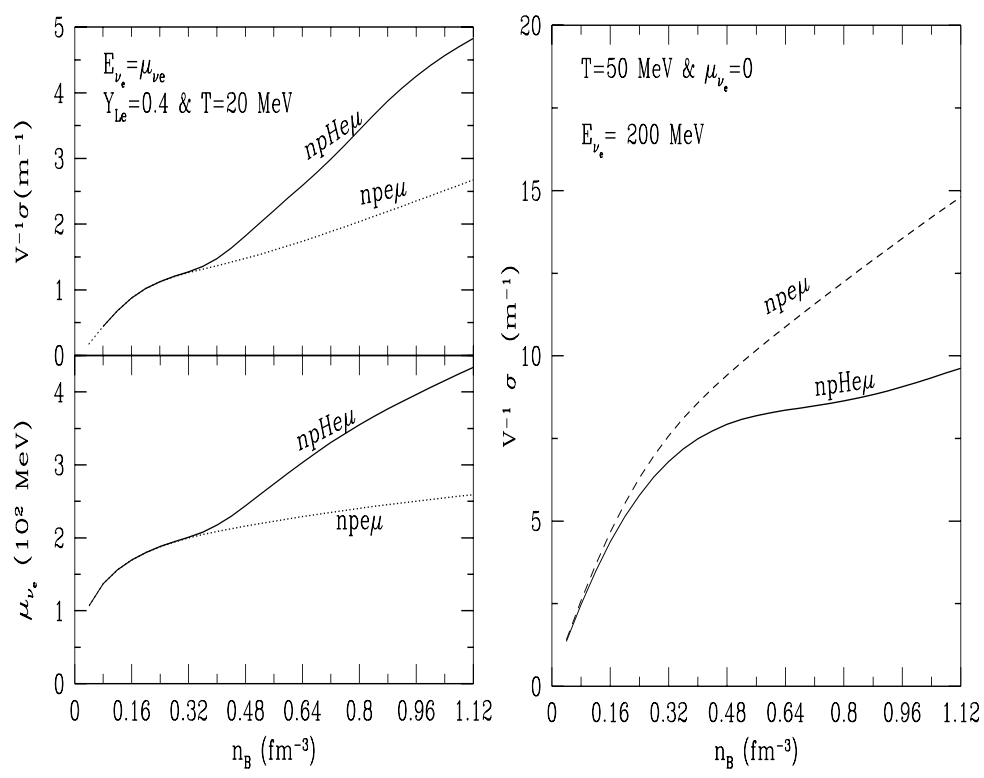

Fig.10. The upper left panel shows neutrino scattering cross sections in neutrino-trapped matter appropriate for the deleptonization phase. The neutrino energy is set equal to the local neutrino chemical potential, which is shown in the lower left panel. The right panel shows cross sections for thermal neutrinos in neutrino-poor matter appropriate for the cooling phase. 